\title{
Pancreatic Mass or Cyst? Diagnostic Dilemma
}

\author{
Jagpal Singh Klair ${ }^{\mathrm{a}}$ Rahman Nakshabendi ${ }^{\mathrm{a}}$ Maheen Rajput ${ }^{\mathrm{b}}$ Henning Gerke ${ }^{\mathrm{a}}$ \\ Rami El-Abiad ${ }^{a}$ \\ aDivision of Gastroenterology and Hepatology, University of lowa Hospitals and Clinics, lowa City, IA, USA; \\ ${ }^{b}$ Department of Radiology, University of lowa Hospitals and Clinics, lowa City, IA, USA
}

\section{Keywords}

Pancreatic divisum · Pancreatitis · Santorinicele $\cdot$ Minor papilla $\cdot$ Sphincterotomy

\begin{abstract}
A santorinicele is a rare anomaly defined as focal cystic dilation of the terminal portion of the dorsal pancreatic duct at the minor papilla. Importantly this anomaly has been suggested as a possible cause of relative stenosis of the minor papilla. This anomaly has been associated with pancreatic divisum and recurrent acute pancreatitis. Magnetic resonance imaging/magnetic resonance cholangiopancreatography and endoscopic ultrasound (EUS) are the main diagnostic modalities. Endoscopic minor papilla sphincterotomy has been shown to improve pain and quality of life in patients with this anomaly presenting with recurrent pancreatitis. We present a case of a single episode of pancreatitis who underwent EUS for evaluation of possible pancreatic mass leading to a diagnosis of santorinicele and complete pancreatic divisum. We attempt to describe this anomaly, diagnostic approach, and management options.
\end{abstract}

\section{KARGER}

(c) 2019 S. Karger AG, Basel

E-Mail karger@karger.com

www.karger.com/ddi

\section{Introduction}

A santorinicele is a focal cystic dilation of the distal dorsal pancreatic duct (duct of Santorini) at the minor papilla. This usually occurs in the setting of pancreatic divisum, although santorinicele without pancreatic divisum has been reported previously. A santorinicele can also be associated with a periampullary diverticulum. We present the case of an elderly male with an attack of pancreatitis attributed to a pancreatic head lesion which was found to be a santorinicele associated with complete pancreatic divisum on endoscopic ultrasound (EUS). It is important to be cognizant of the EUS findings in evaluating the etiology of acute pancreatitis.

\section{Case Presentation}

A 63-year-old otherwise healthy Hispanic male was referred to our gastrointestinal clinic for EUS to evaluate a suspicious pancreatic head mass found on magnetic resonance imaging (MRI). He was recently hospitalized with an episode of acute uncomplicated pancreatitis. Laboratory evaluation on admission showed a lipase of 14,000 U/L with normal complete blood count, comprehensive
Jagpal Singh Klair, MD

Division of Gastroenterology and Hepatology University of Iowa Hospitals and Clinics 200 Hawkins Drive, Iowa City, IA 52242 (USA) E-Mail klairjagpals@gmail.com 


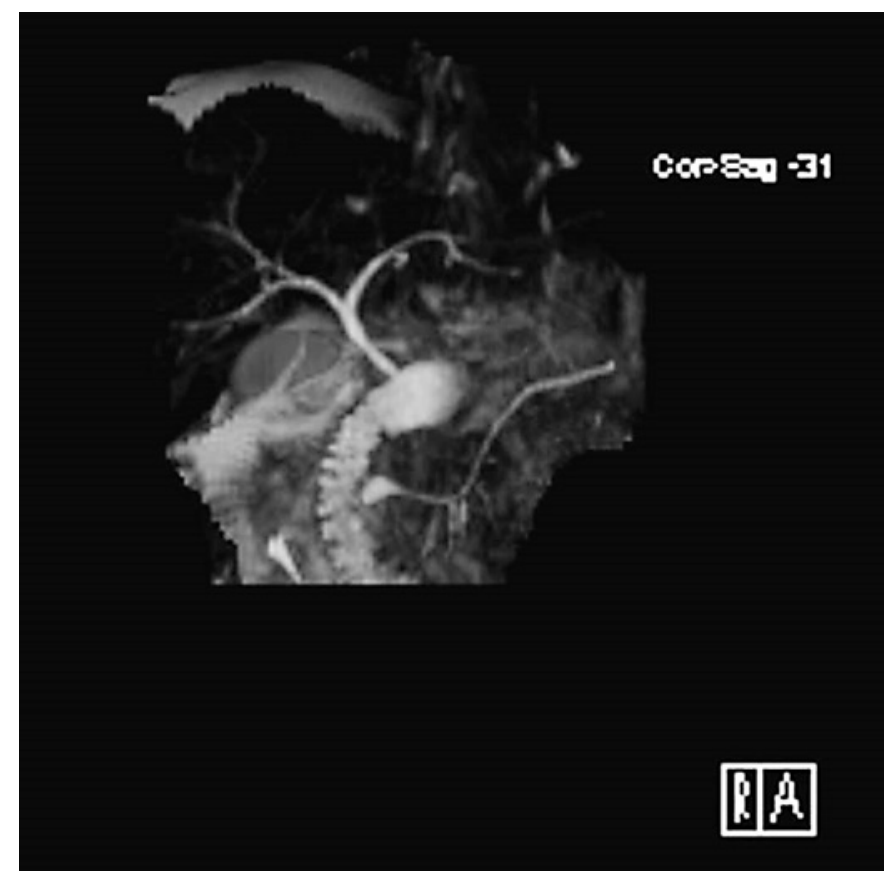

Fig. 1. Magnetic resonance cholangiopancreatography image showing santorinicele and pancreatic divisum.

metabolic panel, triglycerides, and calcium levels. He was on no medications. He denied any history of smoking or alcohol consumption. His family history was negative for pancreatic cancer. He underwent right upper quadrant ultrasound outside the hospital which ruled out gallstones. Abdominal computed tomography with contrast done at an outside hospital revealed acute pancreatitis with mild peripancreatic inflammatory changes, a small ill-defined structure in the pancreatic head, and no common bile duct stone. This was followed by an abdominal MRI at an outside hospital which showed a $2-\mathrm{cm}$ vague area of decreased signal in the pancreatic head suspicious for possible mass, but no obstruction of the pancreatic duct or adjacent adenopathy was noted (Fig. 1, 2 ). The patient had an uneventful hospital course and was discharged 3 days later.

He was referred to our institution for EUS evaluation of the pancreatic head findings. The EUS did not show any pancreatic parenchymal or ductal changes suggesting chronic pancreatitis. In addition, no mass lesions were seen, and the bile duct was of normal caliber, with no cholelithiasis or choledocholithiasis. However, the dorsal pancreatic duct opened into a cystic dilation which measured $6 \mathrm{~mm}$, suggesting presence of a santorinicele. There was no clear communication between the dorsal duct and the ventral pancreas duct consistent with complete pancreatic divisum (Video 1). Endoscopic sphincterotomy of the minor papilla was offered, but the patient preferred watchful waiting, but agreed to proceed in case of recurrent pancreatitis.

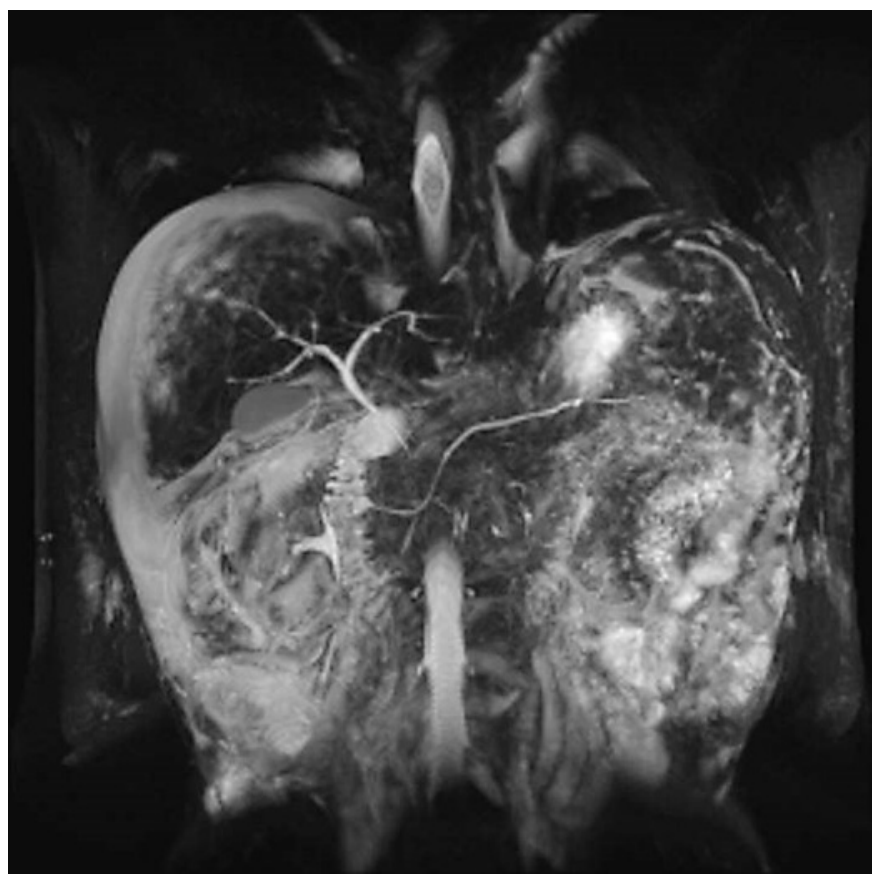

Fig. 2. Coronal $\mathrm{T} 2$ magnetic resonance imaging image showing santorinicele and pancreatic divisum.

\section{Discussion}

In the absence of other causes of pancreatitis and in the setting of pancreatic divisum, a santorinicele can be considered in the differential diagnosis of recurrent acute pancreatitis. The mechanism is likely due to papillary hypertension from a stenotic minor papilla impairing pancreatic juice outflow [1-4]. The diagnosis is usually achieved by MRI/magnetic resonance cholangiopancreatography with accuracy improved by intravenous secretin injection. Endoscopic retrograde cholangiography or EUS can also play a role in the diagnosis or treatment [2, 4]. Our case demonstrates that EUS can provide a minimally invasive diagnostic modality in the diagnosis of a santorinicele if MRI remains nondiagnostic. EUS provides detailed imaging of the pancreatic parenchyma and ductal system without injecting contrast into these ducts which can help in diagnosing early chronic pancreatitis, small pancreatic tumors, and ductal abnormalities. Especially linear echoendoscopes as opposed to radial ones are useful to follow the course of the pancreatic duct. Focal cystic dilation at the terminal portion of the duct of Santorini at the minor papilla, especially in patients with pancreatic divisum, is suspicious for a santorinicele. The duct of Santorini is visualized entering the duodenum proxi- 


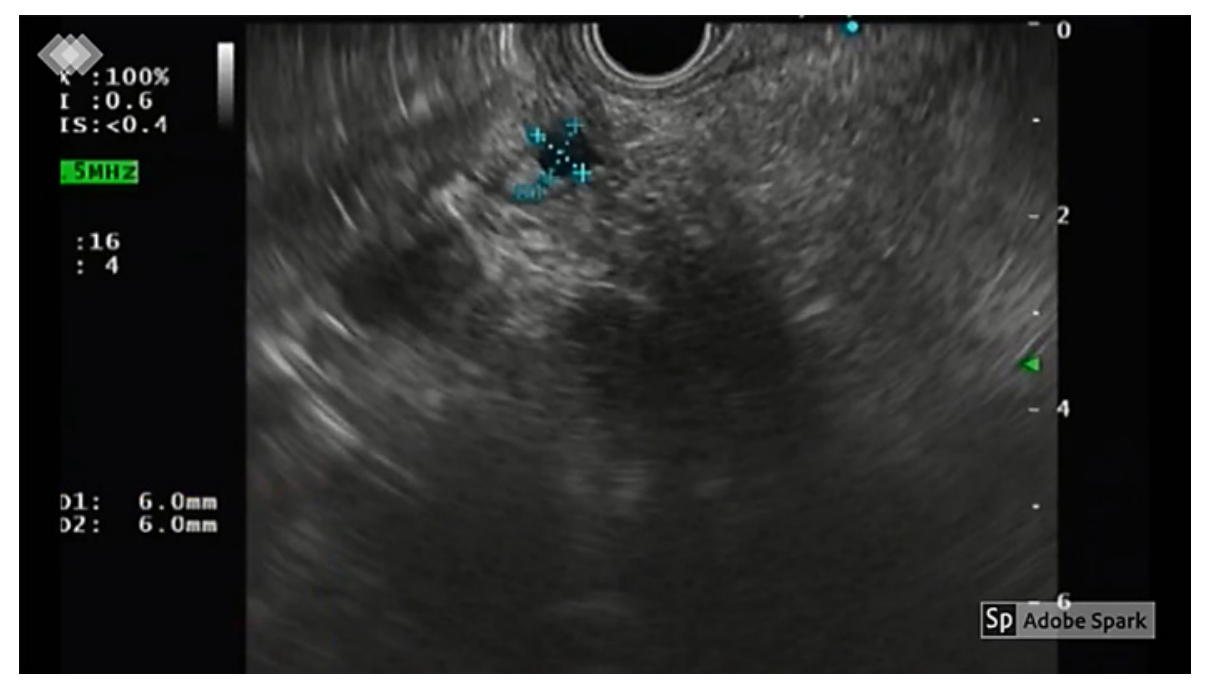

Video 1. Endoscopic ultrasound video and magnetic resonance images of the santorinicele and complete pancreatic divisum with an audio dictation of the case.

mal to the common bile duct. In pancreatic divisum, no connection between the ventral pancreatic duct and the dorsal duct is seen on EUS, and the duct of Santorini is often more prominent than the ventral pancreatic duct because of physiological overload. The diagnosis of pancreatic divisum is excluded if the pancreatic duct can be followed continuously from the major papilla into the pancreatic body crossing the endosonographic border between the hypoechoic ventral anlage and the hyperechoic dorsal pancreas.

As mentioned above, either MRI or EUS can diagnose santorinicele and pancreatic divisum. In this case, we happen to have both. This was an unintentional duplication as this patient was referred for an EUS to evaluate a pancreatic mass noted on an outside MRI of the abdomen. Technical challenges delayed the arrival of the MRI images from the local facility, until a week after completion of the EUS. Upon review of the MRI images, we rescinded the diagnosis of pancreatic mass and confirmed the EUS findings of santorinicele and pancreatic divisum.

Endoscopic minor papilla sphincterotomy (EMPS) has been shown to be effective in reducing the pain and frequency of pancreatitis and improving the quality of life of symptomatic patients with santorinicele and pancreatic divisum [3]. EMPS reduces intraductal pressure and improves pancreatic fluid outflow [2,3]. The indications for endoscopic intervention include recurrent pancreatitis or recurrent pain with elevated pancreatic enzymes [1]. There is a small risk of post-sphincterotomy stenosis that may require repeat sphincterotomy. Pancreatic stent placement is suggested after minor sphincterotomy to reduce the risk of post-sphincterotomy pancreatitis.
Our patient had only one episode of pancreatitis and was asymptomatic and pain free at the time of EUS. Pancreatic enzymes were normal. Management options including EMPS were discussed in detail with the patient, but he elected to pursue the approach of watchful waiting. He agreed however to proceed with intervention should he experience recurrent bouts of pancreatitis. The patient is still doing well 4 months later.

\section{Conclusion}

A santorinicele associated with pancreatic divisum is a rare cause of acute pancreatitis. It may mimic a pancreatic mass on cross-sectional imaging. The diagnosis can be made with linear EUS in a minimally invasive fashion.

\section{Statement of Ethics}

Informed consent was obtained from the patient.

\section{Disclosure Statement}

None of the authors have any conflict of interest to declare.

\section{Author Contributions}

J.S. Klair: literature review, drafting, and editing. R. Nakshabendi: literature review. M. Rajput: literature review and critical revision of the manuscript. $\mathrm{H}$. Gerke: critical review of the manuscript. R. El-Abiad: critical revision of the manuscript. 


\section{References}

1 Crinò SF, Bernardoni L, Conti Bellocchi MC, Malleo G, Manfredi R, Breoni I, et al. Efficacy of Endoscopic Minor Papilla Sphincterotomy for Symptomatic Santorinicele. Clin Gastroenterol Hepatol. 2017 Feb;15(2):303-6.

2 Boninsegna E, Manfredi R, Ventriglia A, Negrelli R, Pedrinolla B, Mehrabi S, et al. Santorinicele: secretin-enhanced magnetic resonance cholangiopancreatography findings before and after minor papilla sphincterotomy. Eur Radiol. 2015 Aug;25(8):2437-44.
3 Lutzak GD, Gluck M, Ross AS, Kozarek RA. Endoscopic minor papilla sphincterotomy in patients with santoriniceles reduces pain and improves quality of life. Dig Dis Sci. 2013 Jul; 58(7):2075-81.

4 Manfredi R, Costamagna G, Brizi MG, Spina S, Maresca G, Vecchioli A, et al. Pancreas divisum and "santorinicele": diagnosis with dynamic MR cholangiopancreatography with secretin stimulation. Radiology. 2000 Nov; 217(2):403-8.
Klair/Nakshabendi/Rajput/Gerke/ El-Abiad 